\title{
Study of the Effects of Exercise and Food Additive Monosodium Glutamate on Life Span Using Caenorhabditis elegans
}

\author{
M.A.F. Sabriya and D.H.H. Munasinghe* \\ Department of Botany, University of Sri Jayewardenepura, Sri Lanka
}

Date Received 02-01-2018

Date Accepted: 21-10-2018

\begin{abstract}
Human health and life span depend on the quality and the quantity of diet. Since it is difficult to evaluate the importance of specific diet that exerts on health and life expectancy using human, a simple model organism Caenorhabditis elegans was used. The main objective of this study was to evaluate the effect of food additive monosodium glutamate (MSG) and exercise on lifespan. Wild type and CB1370 strains of C. elegans were grown in nematode growth medium (NGM) plates seeded with $E$. coli OP50. Separate NGM plates were prepared with different concentrations of MSG and agar. An increased concentration of agar was used to exercise the worm population. The population was scored on daily basis until the whole population was dead. Final data were analyzed using OASIS (Online Application for Survival Analysis) and life span curves were generated. The lowest MSG concentration $(0.05 \mathrm{mM})$ declined the mean life span of $\mathrm{N} 2$ by $13.77 \%(\mathrm{P}<0.05)$. With increasing MSG concentration 5 $\mathrm{mM}, 20 \mathrm{mM}$ and $24 \mathrm{mM}$ the mean life span is further decreased by $16.83 \%(\mathrm{P}<0.05)$. In the exercise gradient experiment although the percentage life span changes were positive and significant, they were not reproducible. Overall the present study suggests that MSG exerts a significant reduction in C. elegans lifespan and the gene daf-2 which is mutated in CB1370 strain has an impact on this negative effect caused by MSG to the life span. Since $C$. elegans share $80 \%$ homology to human, there is a possibility to have a similar effect of MSG on human lifespan and further experiments would reveal the validity of this hypothesis.
\end{abstract}

Key words: exercise, food additive, monosodium glutamate, lifespan

\section{Introduction}

Aging is a degenerative process in nature, which is characterised by a progressive deterioration of cellular components and organelles resulting in mortality. Therefore, when considering total rate of aging, lifespan is the primary end point (Sutphin and Kaeberlein, 2009). Although, the lifespan is largely influenced by random and stochastic degenerative process, it is exclusively influenced by both genetic and environmental factors.

For a long time, use of flavorings, coloring agents and preservatives has been known in the food industries and the excess concentrations of these chemicals are harmful for human health. Monosodium Glutamate (MSG) is such widely used flavor enhancer which elicits a taste described as umami (savory in Japanese language) (Husarova and Ostantnikova, 2013). Due to its enormous use in food industry, individual consumption of MSG is steadily

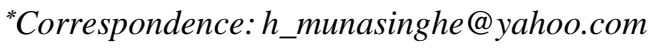

(C) University of Sri Jayewardenepura 
increasing worldwide. There are evidences to show that exceeding levels of MSG accumulation leads to trauma, neural injury, and associated metabolic failures (Paul and Manoj, 2009). Moreover, studies showed that there is an association between obesity and neonatal or maternal administration of high dose of MSG possibly acting on immature neurological mechanism that regulates food intake and energy expenditure (Insawang et al., 2012). Unlike MSG, it is believed that exercise confers powerful health benefits with anticancer, anti-diabetes, anti sarcopenia, anti-cognitive decline and possibly pro immune consequences. Hence, the molecular and cellular mechanism by which exercise improves the health span remains poorly understood (Rahman et al., 2014).

Since it is difficult to evaluate the importance of specific diet and activities that exerts on health and life expectancy using human, nowadays researchers have found a new simple model organism Ceanorhabditis elegans for lifespan researches (Paul and Manoj, 2009). As an existing primitive organism, $C$. elegans shares many of the essential biological characteristics that are the central problems of human biology. Worm is conceived as a single cell, which undergoes a complex process of development starting with embryonic cleavage and grows in to adult through morphogenesis. It exhibits behavior and is capable of rudimentary learning. It produces sperm and eggs, mates and reproduces. After reproduction it gradually ages losses vigor and finally dies (University of Minnesota, 2015). Amazingly, this simple and small multi cellular worm develops from a fertilized egg to an adult just as all human being does (Donald, 1997; Angier, 1995). In the present study, to determine the relationship between exercise and aging process it is necessary to find a way to induce the worm to exercise. Although, $C$. elegans move with an undulatory motion, they have two distinct movements; crawling movement in semi-solid media and swimming in liquid media. However, in exercise experiment it is hypothesized that introducing the nematode to semisolid media with high agar concentration will increase their activity level as they have to put much effort to crawl in the media with higher agar concentration than the lower one (Munasinghe, 2015).

The main objective of this study was to evaluate the effect of food additive monosodium glutamate and exercise in lifespan of $C$. elegans.

\section{Materials and Methods}

Wild type and CB1370 (daf-2(e1370) III) strains of C. elegans worms and the starter culture of $E$. coli OP50 were received from Caenorhabditis Genetic Centre at the University of Minnesota. Nematode growth medium (NGM) was prepared as reference to Siernagle (2006) and seeded with E. coli OP50. Synchronized worm population was obtained as referred in Siernagle 2006, and used in life span assay experiments. Seeded NGM plates (6 $\mathrm{cm})$ were prepared to test three parameters including two different concentrations of MSG $(0.05 \mathrm{mM} \& 24 \mathrm{mM}), 34 \mathrm{~g} / \mathrm{L}$ agar concentration and the control. L4 larvae were transferred from $\mathrm{N} 2$ and $d a f-2$ synchronous cultures in to respective plates using a stereo microscope. Plates were incubated at $15^{\circ} \mathrm{C}$. Live worms on each plate were monitored and transferred in to fresh seeded plates on each day to separate the adult worms from their progeny until the whole worm population was dead. Live worms were counted daily and dead and censored worms were recorded. Worms with internal hatching of eggs, vulva protrusion, vulva rupture, paralysis, uncoordinated movement, burrowed or crawled off the plate, bacterial or fungal contaminants were excluded from the analysis as censored. Worms failed to respond to repeated light prodding on the head and tail were scored as dead. Since the life span of N2 is 
affected by MSG and exercise than that of CB1370, MSG concentration gradient and exercise gradient were tested on $\mathrm{N} 2$ worms.

Seeded NGM plates were prepared with different MSG concentrations $(0.01,0.05$, $0.1,1,5,10,20$ and $24 \mathrm{mM}$ ) including a control. Above life span experiment was done with N2 synchronous culture and the worm population was scored as explained earlier. Similarly, seeded NGM plates were prepared with different agar concentrations $(17,20,25,30,33$ and $\left.35 \mathrm{gL}^{-1}\right)$. Life span experiment was repeated with $\mathrm{N} 2$ synchronous culture and the worm population was scored. Final data were analyzed using OASIS (Online Application for the Survival Analysis of Life Span Assays) and the life span curves were generated for each test condition. P values were calculated compared to control using Log-Rank Test.

\section{Results}

Based on the results obtained, $0.05 \mathrm{mM}$ and $24 \mathrm{mM}$ MSG concentrations decreases the mean life span of $\mathrm{N} 2$ whereas, it increases the mean life span of daf-2 strain (Table 3.1). When comparing the effect of exercise between $\mathrm{N} 2$ and daf-2 strain, the results showed that the mean life span of daf-2 strain is further decreased than that of $\mathrm{N} 2$ at $34 \mathrm{~g} / \mathrm{L}$ agar concentration. Although it was not significant, as it is believed that exercise positively impact the lifespan while monosodium glutamate has a negative impact on life span based on previous findings, wild type worms were selected for further studies on MSG and exercise gradient experiments.

According to the results obtained the mean life span of $\mathrm{N} 2$ is significantly decreased with increasing MSG concentration $(\mathrm{P}<0.05)$ (Table 3.2). Although the mean life span reduction is not directly proportional to MSG concentration the mean life span values obtained for different MSG concentrations are significantly lower than the mean life span of control N2 worms $(\mathrm{P} \leq 0.05)$ (Fig. 1).

Table 3.1: Comparison of the effect of MSG concentration and exercise between N2 and CB1370 strain\% change in life span were calculated against the same control data.

\begin{tabular}{lcccccc}
\hline \multirow{2}{*}{ Test } & \multicolumn{2}{c}{$\begin{array}{c}\text { Mean life span at } 95 \% \\
\text { C.I }\left(\text { at } 15^{\circ} \text { C) }\right.\end{array}$} & \multicolumn{2}{c}{ \% Change in life span } & \multicolumn{2}{c}{$\begin{array}{c}\text { Age at }>90 \% \text { mortality } \\
\text { was observed }\end{array}$} \\
\cline { 2 - 7 } & $\mathrm{N} 2$ & $\mathrm{CB} 1370$ & $\mathrm{~N} 2$ & $\mathrm{CB} 1370$ & $\mathrm{~N} 2$ & $\mathrm{CB} 1370$ \\
\hline $0.05 \mathrm{mM}$ MSG & $14.66 \pm 1.75$ & $18.28 \pm 1.66$ & $-24.63 \%$ & $+0.27 \%$ & 25 & 29 \\
$24 \mathrm{mM}$ MSG & $15.23 \pm 1.66$ & $19.24 \pm 2.02$ & -21.750 & $+5.54 \%$ & 29 & 29 \\
$34 \mathrm{~g} / \mathrm{L}$ & $17.63 \pm 1.83$ & $14.72 \pm 1.44$ & $-9.36 \%$ & $-19.25 \%$ & 29 & 25 \\
Control & $19.45 \pm 1.59$ & $18.23 \pm 2.02$ & - & - & 29 & 29
\end{tabular}

Increase and decrease in life span was indicated as (+) and (-) signs respectively.

Table 3.2: Lifespan values of wild type C. elegans (N2) at different concentrations of MSG.

\begin{tabular}{lcccc}
\hline $\begin{array}{c}\text { Test/MSG } \\
\text { Concentration }\end{array}$ & $\begin{array}{c}\text { Mean life span at } \\
95 \% \text { C.I }\left(\text { at } 15^{\circ} \mathrm{C}\right)\end{array}$ & $\begin{array}{c}\text { Age at }>90 \% \\
\text { mortality }\end{array}$ & $\begin{array}{c}\% \text { change in life } \\
\text { span }\end{array}$ & $\begin{array}{c}\text { Log-Rank Test P } \\
\text { values }\end{array}$ \\
\hline $0.05 \mathrm{mM}$ & $18.60 \pm 0.90$ & 29 & $-13.77 \%$ & $0.0237^{*}$ \\
$5 \mathrm{mM}$ & $17.32 \pm 0.85$ & 26 & $-19.70 \%$ & $0.0024^{*}$ \\
$20 \mathrm{mM}$ & $17.64 \pm 0.85$ & 25 & $-18.22 \%$ & $0.0016^{*}$ \\
$24 \mathrm{mM}$ & $17.94 \pm 0.75$ & 21 & $-16.83 \%$ & $0.0051^{*}$ \\
Control & $21.57 \pm 0.94$ & 32 & 0 & - \\
\hline
\end{tabular}

$p$ values and \% change in life span were calculated against the same control data; increase and decrease in life span was indicated as (+) and (-) signs respectively. * indicated $p<0.05$. 
As the results obtained for the exercise gradient test, the mean life span of $\mathrm{N} 2$ is significantly increased at $33 \mathrm{~g} / \mathrm{L}$ and $35 \mathrm{~g} / \mathrm{L}$ agar concentrations $(\mathrm{P}<0.05)$ compared to the control group (Table 3.3, Fig. 2). However these significant changes in the life span were not reproducible.

Table 3.3: Life span values of wild type $C$. elegans (N2) at different concentrations of agar.

\begin{tabular}{ccccc}
\hline $\begin{array}{c}\text { Test/Agar } \\
\text { Concentration, g/L }\end{array}$ & $\begin{array}{c}\text { Mean life span at } \\
\text { 95\% C.I (at 15 } 5^{\circ} \text { C }\end{array}$ & $\begin{array}{c}\text { Age at }>90 \% \\
\text { mortality }\end{array}$ & $\begin{array}{c}\text { \%change in life } \\
\text { span }\end{array}$ & $\begin{array}{c}\text { Log-Rank Test } p \\
\text { values }\end{array}$ \\
\hline 20 & $21.82 \pm 1.17$ & 38 & $+3.36 \%$ & 0.8845 \\
25 & $21.29 \pm 1.21$ & 42 & $+0.85 \%$ & 0.9264 \\
30 & $21.51 \pm 1.71$ & 43 & $+1.89 \%$ & 0.4972 \\
33 & $25.33 \pm 1.84$ & 49 & $+19.99 \%$ & $0.0261^{*}$ \\
35 & $35.30 \pm 2.19$ & 56 & $+67.22 \%$ & $5.9 \mathrm{e}^{-08 *}$ \\
Control (17) & $21.11 \pm 1.19$ & 39 & 0 & - \\
\hline
\end{tabular}

$p$ values and \% change in life span were calculated against the same control data; increase and decrease in life span was indicated as (+) and (-) signs respectively. * indicates $p<0.05$.

(A)

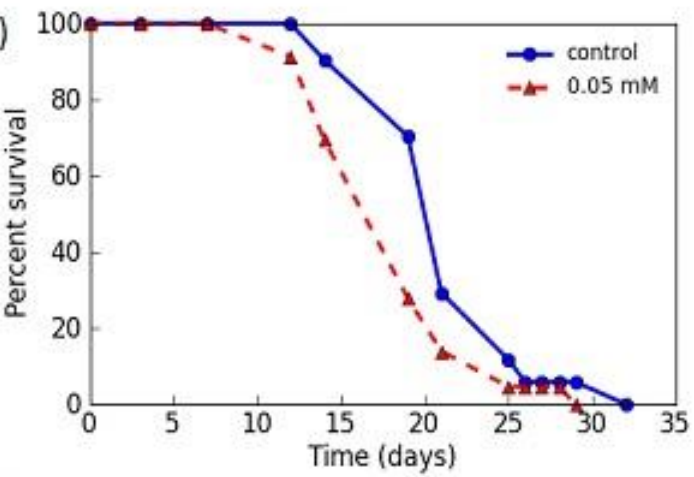

(C)

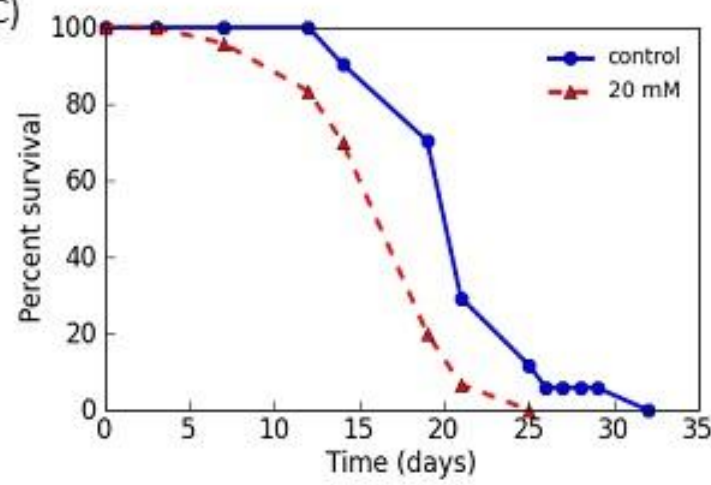

(B)

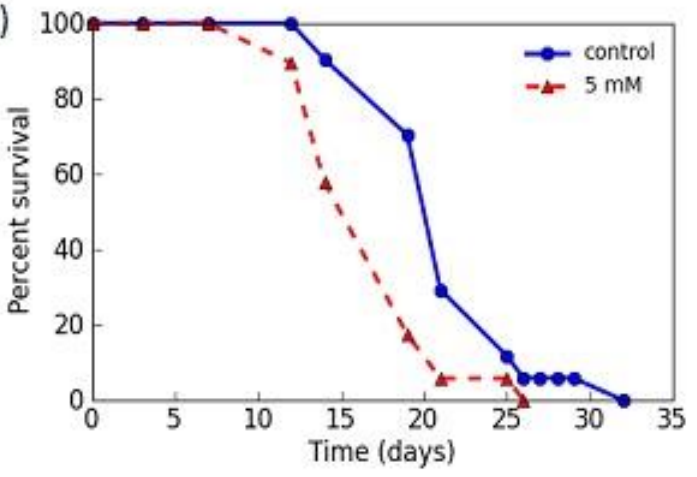

(D)

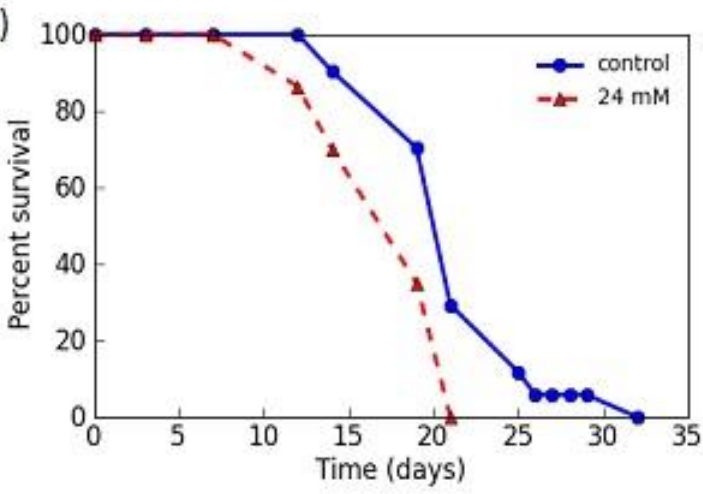

Figure 1: Life span curves of $C$. elegans wild type at different MSG concentrations $(\mathrm{P} \leq 0.05)$. (a) $0.05 \mathrm{mM}$; (b) $5 \mathrm{mM}$; (c) $20 \mathrm{mM}$; (d) $24 \mathrm{mM}$ MSG concentrations 

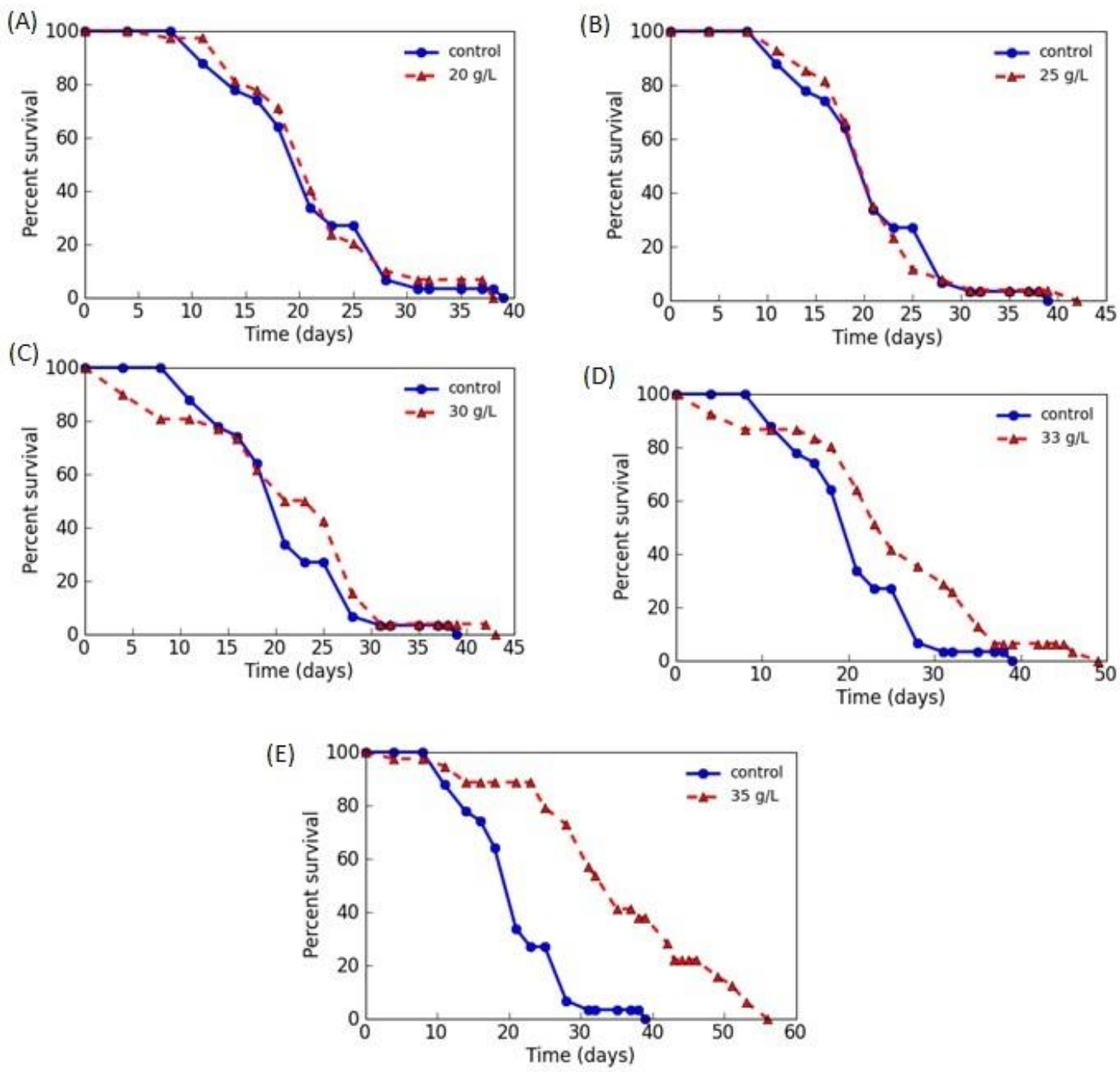

Figure 2: Life span curves of C. elegans wild type (N2) at different exercise gradient. (a) $20 \mathrm{~g} / \mathrm{L}$; (b) $25 \mathrm{~g} / \mathrm{L}$; (c) $30 \mathrm{~g} / \mathrm{L}$; (d) $33 \mathrm{~g} / \mathrm{L}$; (e) $35 \mathrm{~g} / \mathrm{L}$ agar concentrations

\section{Discussion}

The results obtained for the effect of MSG in the life span of $C$.elegans showed a significant reduction in the mean life span of $\mathrm{N} 2$ worms with increasing MSG concentration. Although the reduction is not directly proportional to the concentration of MSG, it suggests that there is a great impact of MSG in reduction of $C$. elegans life span. Considering the results obtained for both $\mathrm{N} 2$ and daf-2 strains, the mean life span of N2 is substantially influenced by MSG. This indicates that the MSG affects lifespan via gene daf-2. In a study conducted by Paul and Manoj in 2009, the toxicity of four food additives was evaluated using C. elegans.

In the study the mortality after 24 hours was adopted as the end point of toxicity and the $\mathrm{LC}_{50}$ values were calculated. The final results showed that the toxicity was in order of Tannic acid>Propyl gallate>Thiourea $>$ MSG. However it is concluded, that the usage of food 
additives in higher concentrations are harmful for human health. Over the past decades, large number of studies has been carried out on the harmful and deleterious effects caused by MSG apart from lifespan assay, using model organisms. A study showed that following the administration of MSG to neonatal rats causes reproductive deficits along with the stunted growth and reduced testis (Pizzi et al., 1977). A following study conducted by the same group of the researchers, revealed that the MSG treated showed increased body weight and decreased pituitary, thyroid, ovary and testis (Pizzi et al. 1977). Obesity is one of the most common problems related to lifestyle nowadays. It is a key risk factor in the development of many chronic diseases. Studies show evidence that people who eat more MSG are more likely to be overweight. In a previous study it was concluded that MSG induces increased lipid storage in C. elegans through AMPK expression by directly activating hypothalamic AMPK, which in turn induce obesity. Moreover, it was reported that MSG may play a role in the development of neurodegenerative diseases by decreasing the neurological function (Sayana 2015). Following a study it was suggested that MSG treatment on mice induces obesity and diabetes with steatosis and steatohepatitis resembling human NAFLD and NASH with pre neoplastic lesions. Further it was concluded that the safety profile of MSG must be reexamined and potentially withdrawn from the food chain (Nakashini et al., 2008).

Exercise and physical activities confer powerful health benefits are hard to ignore. Regardless of age, sex or physical ability everyone benefits from exercise in anti-cancer, antidiabetes, anti-sarcopenia, anti-cognitive decline, and possibly pro immune consequences (Rahman et al., 2014). This gives the motivation to study the relationship between exercise and the aging process. In exercise gradient experiment it showed a significant increase in the mean life span of N2 worms. Although, these results were not reproducible the percentage life span change in the reproducibility test was all positive. This suggests that apart from the influence of exercise in the extension of worm life span there may be other factors contributing towards lifespan.

In a previous study (Rahman et al., 2014) it was reported that swim induced exercise using a microfluidic device enhances the median life span of C. elegans. Furthermore, it was concluded that muscular force of $C$. elegans increases with the developmental stage and the swim train may have greater impact on the animal health span (Rahman et al., 2014). However another report (Voorhies and Ward 1999) suggests that to allow the animal to develop a normal aging phenotype the study organism must be maintained under optimal environmental condition as presumed. Whereas, the $C$. elegans can be grown and maintain in a liquid culture, as it is a non-aquatic, terrestrial nematode in the liquid culture, worms are known to be grown much more slowly, have an altered morphology, cannot mate and have reduced fertility. Therefore, it makes difficult to determine whether the worms undergo normal aging (Voorhies and Ward 1999). Studies showed that apart from the factors discussed above, there are two environmental factors that strongly influence $C$. elegans longevity. They are food availability and growth temperature. When worms are starved, young larvae enter into a dormant state called dauer larvae, capable of moving. These dauer larvae have unique morphology and enter a period of dormancy with reduced activity and metabolic rate. They can survive for about months and this time period is additive to their total longevity. As in all ectotherms it was reported that $C$. elegans longevity is negatively correlated with its growth 
temperature. It is concluded that the wild type worms grown at low temperature live about four times longer than their high temperature counterparts, and the life span of the worm grown at low temperature is approximately as long as the long lived mutant. It was thought that the large effect of temperature in longevity is caused by the influence of temperature on metabolic rate (Voorhies and Ward 1999)

This study concludes that, food additive Monosodium Glutamate exerts a significant reduction in C. elegans life span via DAF-2 insulin like receptor. However, exercise, which is believed to confer powerful health benefits, neither increased nor decreased the mean life span. These findings could lead to future detailed analysis combining genetics and biochemistry. Where it opens a wide avenue to investigate the systemic coordination of aging up to their molecular and genetic level, and to reveal how this leads to healthy aging.

\section{Acknowledgement}

This project was funded by the grants of University of Sri Jayewardenepura (Grant No. ASP/06/RE/SCI/2013/03). C. elegans strains used in this study were provided by the Caenorhabditis Genetic Centre at the University of Minnesota, St. Paul. All the laboratory works were carried out at the Department of Botany, and the microscopic facility was obtained from Department of Parasitology, University of Sri Jayewardenepura. The support of Prof. F. Falciani, University of Liverpool, UK, Prof. R. C. May, University of Birmingham, UK and Dr. S. Hayward, University of Birmingham, UK are also acknowledged.

\section{References}

Angier N. 1995. The beauty of the beastly: new views on the nature of life. Boston, MA; Houghton Mifflin.

Donald D. L. 1997. C. elegans II. New York, NY; Cold Spring Harbor Laboratory Press.

Husarova V., Ostatnikova D. 2013. Monosodium glutamate toxic effects and their implications for human intake: A review. JMED Research 2013: 1-12.

Insawang T., Selmi C., Cha'on U., Pethlert S., Yongvanit P., Areejitranusorn P., Boonsiri P., Khampitak T., Tangrassameeprasert R., Pinitsoontorn C., Prasongwattana V. 2012. Monosodium glutamate (MSG) intake is associated with the prevalence of metabolic syndrome in a rural Thai population. Nutrition and Metabolism, 9(1): 1.

Munasinghe D.H.H. 2015. An integrative approach to understanding effects of valproate in C. elegans http://etheses.bham.ac.uk/6336/, [Accessed on 28 November 2016].

Nakanishi Y., Tsuenyama K., Fujimoto M., Salunga T.L., Nomoto K., An J.L., Takano Y., lizuka S., Nagata M., Suzuki W., Shimada T., Aburada M., Nakano M., Selmi C., Gershwin M.E. 2008. Monosodium glutamate (MSG): A villain and promoter liver inflammation and Dysplasia. Journal of Autoimmun, 30(1-2): 42-50.

Pizzi W.J., Barnhart J.E., Fanslow D.J. 1977. Monosodium glutamate administration to the newborn reduces reproductive ability in female and male mice. Science, 196(4288): 452-454.

Paul S. P., Manoj S. 2009. Screening of food additives on model organism Caenorhabditis elegans. Journal of Industrial pollution Control, 25(2): 1-6.

Rahman M., Burke D., Royal M.A. Gefter L., Chang C., Blawzdziewicz J., Driscoll M, Vanapalli S.A., (2012). Exercise improves strength and confers extended health span on swim-trained C. elegans. Texas Tech University; Obesity Research Cluster Annual Meeting and Networking Event, 9-10. 
Sayana R. 2015. Discovery of MSG as an Inducing factor of lipid storage through expression of AMPK-activated protein kinase. California State Science fair.

Stiernagle T. 2006. Maintenance of C. elegans. Research Community, WormBook.

Stupin G.L., Kaeberlein M. 2009. Measuring Caenorhabditis elegans lifespan on solid media. Journal of Visual Experiments, 27:1-7.

University of Minnesota. 2015. What is C. elegans?;What is Caenorhabditis elegans and why work on it? An introduction for those unfamiliar with "The Worm" http://cbs.umn.edu/ cgc/what-c-elegans [Accessed on 28 November 2016].

Van Voorhies W.A., Ward S. 1999. Genetic and environmental conditions that increase longevity in Caenorhabditis elegans decrease metabolic rate. Proceedings of the National Academy of Sciences, 96(20):11399-11403. 